\title{
Evidence-based clinical practice guidelines for nonalcoholic fatty liver disease/nonalcoholic steatohepatitis
}

\author{
Sumio Watanabe $\cdot$ Etsuko Hashimoto $\cdot$ Kenichi Ikejima $\cdot$ Hirofumi Uto $\cdot$ Masafumi Ono \\ Yoshio Sumida - Masataka Seike - Yoshiyuki Takei - Tetsuo Takehara $\cdot$ Katsutoshi Tokushige • \\ Atsushi Nakajima $\cdot$ Masashi Yoneda $\cdot$ Toshiji Saibara $\cdot$ Goshi Shiota $\cdot$ Isao Sakaida Makoto Nakamuta \\ Toshihiko Mizuta $\cdot$ Hirohito Tsubouchi $\cdot$ Kentaro Sugano $\cdot$ Tooru Shimosegawa
}

Received: 12 December 2014/ Accepted: 25 December 2014/Published online: 24 February 2015

(C) Springer Japan 2015

\begin{abstract}
Nonalcoholic fatty liver disease (NAFLD) is currently the most common cause of chronic liver disease in industrialized countries worldwide, and has become a serious public health issue not only in Western countries but also in many Asian countries including Japan. Within the wide spectrum of NAFLD, nonalcoholic steatohepatitis (NASH) is a progressive form of disease, which often develops into liver cirrhosis and increases the risk of hepatocellular carcinoma. In turn, a large proportion of NAFLD/NASH is the liver manifestation of metabolic syndrome, suggesting that NAFLD/NASH plays a key role in the pathogenesis of systemic atherosclerotic diseases. Currently, a definite diagnosis of NASH requires liver biopsy, though various noninvasive measures are under development. The mainstays of prevention and treatment of NAFLD/NASH include dietary restriction and exercise; however, pharmacological approaches are often necessary.
\end{abstract}

The original version of this article appeared in Japanese as "NAFLD/ NASH, Shinryo Guidelines 2014," from the Japanese Society of Gastroenterology (JSGE), published by Nankodo, Tokyo, 2014. Please see the article on the standards, methods, and process of developing the guidelines (doi:10.1007/s00535-014-1016-1). These guidelines are published jointly in the Journal of Gastroenterology and Hepatology Research. The members of the Working Committee are listed in the Appendix in the text.

S. Watanabe $(\varangle) \cdot$ E. Hashimoto $\cdot$ K. Ikejima $\cdot$ H. Uto

M. Ono · Y. Sumida $\cdot$ M. Seike · Y. Takei · T. Takehara

K. Tokushige $\cdot$ A. Nakajima $\cdot$ M. Yoneda - T. Saibara .

G. Shiota · I. Sakaida · M. Nakamuta · T. Mizuta ·

H. Tsubouchi · K. Sugano - T. Shimosegawa

Guidelines Committee for creating and evaluating the

"Evidence-based clinical practice guidelines for nonalcoholic fatty liver disease/nonalcoholic steatohepatitis", the Japanese Society of Gastroenterology (JSGE), K-18 Building 8F, 8-9-13

Ginza, Chuo, Tokyo 104-0061, Japan

e-mail: sumio@juntendo.ac.jp
Currently, vitamin $\mathrm{E}$ and thiazolidinedione derivatives are the most evidence-based therapeutic options, although the clinical evidence for long-term efficacy and safety is limited. This practice guideline for NAFLD/NASH, established by the Japanese Society of Gastroenterology in cooperation with The Japan Society of Hepatology, covers lines of clinical evidence reported internationally in the period starting from 1983 to January 2012, and each clinical question was evaluated using the GRADE system. Based on the primary release of the full version in Japanese, this English summary provides the core essentials of this clinical practice guideline comprising the definition, diagnosis, and current therapeutic recommendations for NAFLD/NASH in Japan.

Keywords NAFLD/NASH guideline · Treatment

\section{Introduction}

Changes in diet and lifestyle have led to a dramatic increase in the prevalence of obesity and metabolic syndrome in Western countries and many Asian countries. This has resulted in a significant increase in the incidence of nonalcoholic fatty liver disease (NAFLD) [1-6]. NAFLD consists of two clinical entities: nonalcoholic fatty liver (NAFL) and nonalcoholic steatohepatitis (NASH) [7-11]. NAFLD is currently the most common cause of chronic liver disease and has become an important public health issue. An evidence-based clinical practice guideline for NAFLD/NASH is needed. However, there is currently insufficient evidence available for a clinical practice guideline. Moreover, there is no clear consensus regarding the alcohol intake threshold to define "nonalcoholic liver disease," to exclude other liver diseases from NAFLD, and to 
histologically define steatohepatitis. Accordingly, definitions of NAFLD/NASH for this clinical practice guideline are provided first. This guideline summarizes reports from 1983 to the end of January 2012.

\section{Definitions}

NAFLD is characterized by evidence of hepatic steatosis either by imaging or histology and appropriate exclusion of other liver diseases such as alcoholic liver disease. Epidemiological studies have shown that alcoholic liver disease can occur when the daily alcohol intake exceeds $20 \mathrm{~g}$ in women and $30 \mathrm{~g}$ in men $[12,13]$. Therefore, NAFLD is diagnosed when the alcohol consumption is lower than the aforementioned amounts. NAFLD is associated with obesity, diabetes mellitus, dyslipidemia, and hypertension, and is considered the hepatic manifestation of metabolic syndrome. NAFLD is histologically characterized by macrovesicular steatosis and further categorized into NAFL and NASH. NAFL is mostly a benign, nonprogressive clinical entity, while NASH can progress to cirrhosis or even hepatocellular carcinoma (HCC). NASH is histologically characterized by hepatic steatosis associated with evidence of liver cell injury (ballooning degeneration) and inflammation [7-11].

Additional comments:

- NAFLD has several etiologies aside from excessive caloric intake, including endocrine diseases, severe malnutrition, and drug side effects.

- Histologic characteristics of NASH are lost with progression to end-stage cirrhosis; this is called "burned-out" NASH.

\section{Epidemiology}

Incidence and prevalence of NAFLD/NASH

in the general population

The reported prevalence of NAFLD varies widely depending on the population studied and the definition used [14-18]. Age, gender, and ethnic differences are present in both the prevalence and severity of NAFLD/NASH [1921]. These differences are also seen in the prevalence of obesity and metabolic syndrome. The prevalence of NAFLD is $20-40 \%$ in Western countries and $12-30 \%$ in Asian countries. Annual health checkup data showed that 9-30 \% of Japanese adults have ultrasonography (US)-diagnosed NAFLD. NASH is diagnosed in 10-20\% of cases of NAFLD. The estimated prevalence of NASH is approximately $3-5 \%$ worldwide. NAFLD/NASH is predominantly diagnosed in middle-aged men and in postmenopausal women.

Prevalence of NAFLD/NASH in high-risk groups, in chronic liver diseases, and in the pediatric population

The prevalence of NAFLD increases with the severity of risk factors. It is $10-20 \%$ in non-obese individuals, approximately $50 \%$ in those with a body mass index (BMI) more than $25 \mathrm{~kg} / \mathrm{m}^{2}$ but less than $30 \mathrm{~kg} / \mathrm{m}^{2}$, and approximately $80 \%$ in those with a BMI over $30 \mathrm{~kg} / \mathrm{m}^{2}$ [14]. The prevalence of NAFLD is approximately $50 \%$ in patients with type 2 diabetes. High serum cholesterol levels, low serum high-density lipoprotein, and high serum triglyceride levels are also risk factors for NAFLD. These risk factors for NAFLD are likewise risk factors for metabolic syndrome.

The true prevalences of NASH in cirrhosis and in HCC are difficult to evaluate because histological evaluation is required for the diagnosis of $\mathrm{NASH}$, introducing selection bias. Further, characteristic features of NASH disappear in the end stage of cirrhosis ("burned-out" NASH), making diagnosis impossible [22, 23]. According to a nationwide retrospective survey on the etiology of liver cirrhosis, NAFLD accounts for $2.1 \%$ of all cirrhosis in Japan [24]. NAFLD/NASH-related HCC accounts for 10-24 \% of all HCC in Western countries and 2-5\% in Japan [25]. Pediatric NAFLD is also related to obesity and metabolic syndromes. The prevalence of NAFLD in the pediatric population is estimated to be $3 \%$ and may increase with age. In pediatric NAFLD patients, genetic testing may be considered [26-29].

\section{Clinical features}

NAFLD patients are usually asymptomatic until the condition progresses to liver cirrhosis. Therefore, NAFLD is often detected during routine health checkups or clinical visits for other diseases. Most patients with NAFLD are obese, and many have type 2 diabetes, dyslipidemia and/or hypertension. NAFLD is no longer considered to be a primary liver disease, but rather a component of metabolic syndrome.

NAFLD could be the result of insulin resistance. The development of NASH is thought to initiate from basal steatosis as the "first hit," followed by a "second hit" that induces necroinflammation. This hypothesis is called the "two-hit theory" [30]. The second hit can arise from oxidative stress, especially mitochondrial stress, insulin resistance, inflammatory cytokines, impaired autophagy, or deleterious microbiota, etc. Recently, a new hypothesis to explain the pathogenesis of NASH was reported by Tilg 
and Moschen [31], namely the "multi-parallel hit" hypothesis. This hypothesis, based on reports that endoplasmic reticulum stress and cytokine-mediated stress can induce steatosis as well as necroinflammation, suggests that multiple hits act together in parallel in the development of NASH. Steatosis should therefore be considered part of the liver's early "adaptive" response to stress, rather than the first hit in disease progression.

Familial clustering and ethnic differences in susceptibility indicate that genetic factors may be important risk determinants for development and progression of NAFLD/ NASH [32-36]. Variation in the enzyme adiponutrin (patatin-like phospholipase domain-containing protein 3; PNPLA3) is a major genetic determinant of hepatic steatosis and fibrosis [33-35].

NAFLD/NASH unrelated to obesity or metabolic syndrome

NAFLD/NASH can be induced by drugs, surgery, or endocrine diseases. Drug-induced mitochondrial damage, obesity, type 2 diabetes, and/or dyslipidemia cause NAFLD/NASH [37]. Many patients develop NAFLD/ NASH after pancreaticoduodenectomy. These patients are neither obese nor insulin resistant. Although the main etiology of NAFLD/NASH after pancreatic resection remains unclear, intensifying pancreatic enzyme supplementation may be useful [38]. Patients with short bowel syndrome also develop NAFLD/NASH due to severe malnutrition. Many endocrine diseases such as hypothyroidism, hypopituitarism, hypogonadism, and polycystic ovary syndrome are risk factors for the development of NAFLD/ NASH [39-43]. These endocrine diseases can lead to obesity, insulin resistance, and/or dyslipidemia, which can in turn result in NAFLD.

\section{Diagnosis}

\section{Diagnostic strategies for NAFLD/NASH}

Diagnosis of NAFLD is based on the following three criteria: detection of steatosis either by imaging or histology, the exclusion of alcoholic liver disease, and appropriate exclusion of other liver diseases such as viral hepatitis, autoimmune liver diseases, and metabolic or hereditary liver diseases [10, 11] (Fig. 1). The criterion for "nonalcoholic" is that daily alcohol consumption be lower than $20 \mathrm{~g}$ in women and $30 \mathrm{~g}$ in men. NASH is diagnosed from a liver biopsy on the basis of the presence of steatohepatitis.

Blood chemistry shows mild elevation of transaminases. Serum transaminase levels are helpful in screening for
NAFLD, but do not indicate NAFLD severity. It is important to consider that a substantial number of NAFLD patients have normal transaminase levels [44, 45]. In patients with NAFLD, it is reasonable to assess the patient for metabolic syndrome, glucose intolerance, and dyslipidemia, because NAFLD may indicate the presence of these diseases. In stage 3 fibrosis, fibrosis markers such as hyaluronic acid are elevated, and in the cirrhotic stage, platelet counts are low with evidence of liver dysfunction such as elevation of serum bilirubin and ammonia [46-49].

A liver biopsy has several drawbacks. It is an expensive and invasive procedure and there is potential for sampling error and variability in interpretation by pathologists. Moreover, given the high prevalence of NAFLD, liver biopsy is poorly suited as a diagnostic test for NASH. Liver biopsies may only be advocated in NAFLD patients who are considered to be at increased risk for NASH with advancing fibrosis, or are suspected of having coexisting chronic liver diseases where there is a need to distinguish NASH from other chronic liver diseases.

Noninvasive assessment of NASH and advanced fibrosis in NAFLD

There has been intense interest in the development of biochemical markers and scoring systems for predicting NASH or fibrosis [50-53]. Circulating levels of cytokeratin-18 (CK18) fragments have been investigated most extensively as a novel biomarker for the diagnosis of NASH [50]. However, there are no practically useful surrogate markers for diagnosing NASH. The NAFLD fibrosis score [52] and the Enhanced Liver Fibrosis (ELF) panel [53] are scoring systems for predicting fibrosis. In general, the accuracy of these scoring systems for the diagnosis of mild fibrosis is weak. The NAFLD fibrosis score is a widely validated scoring system for predicting the severity of fibrosis. It is based on six readily assessable clinical variables that include platelet count, albumin, and the aspartate transaminase/alanine transaminase (AST/ALT) ratio, and is calculated using a published formula (http:// nafldscore.com).

Imaging modalities

Imaging modalities can detect fatty changes. Abdominal US is currently the most common method for assessing hepatic steatosis [54]. Computed tomography and magnetic resonance imaging (MRI) seem to be more objective and more sensitive techniques for the quantification of steatosis, but MRI is still less widely available and much more expensive $[55,56]$. Unfortunately, no imaging modality is useful for the diagnosis of NASH. US-based transient 
Fig. 1 Flowchart for diagnosis of NAFLD/NASH. Patients with hepatic steatosis and/or abnormal liver biochemistries are tested as follows: First, check hepatitis B virus surface antigen (HBsAg), antihepatitisC virus (anti-HCV), and autoantibodies for exclusion of other liver diseases; second, check alcohol consumption to differentiate alcoholic andnonalcoholic liver diseases; third, diagnose NAFL or NASH on the basis of histology

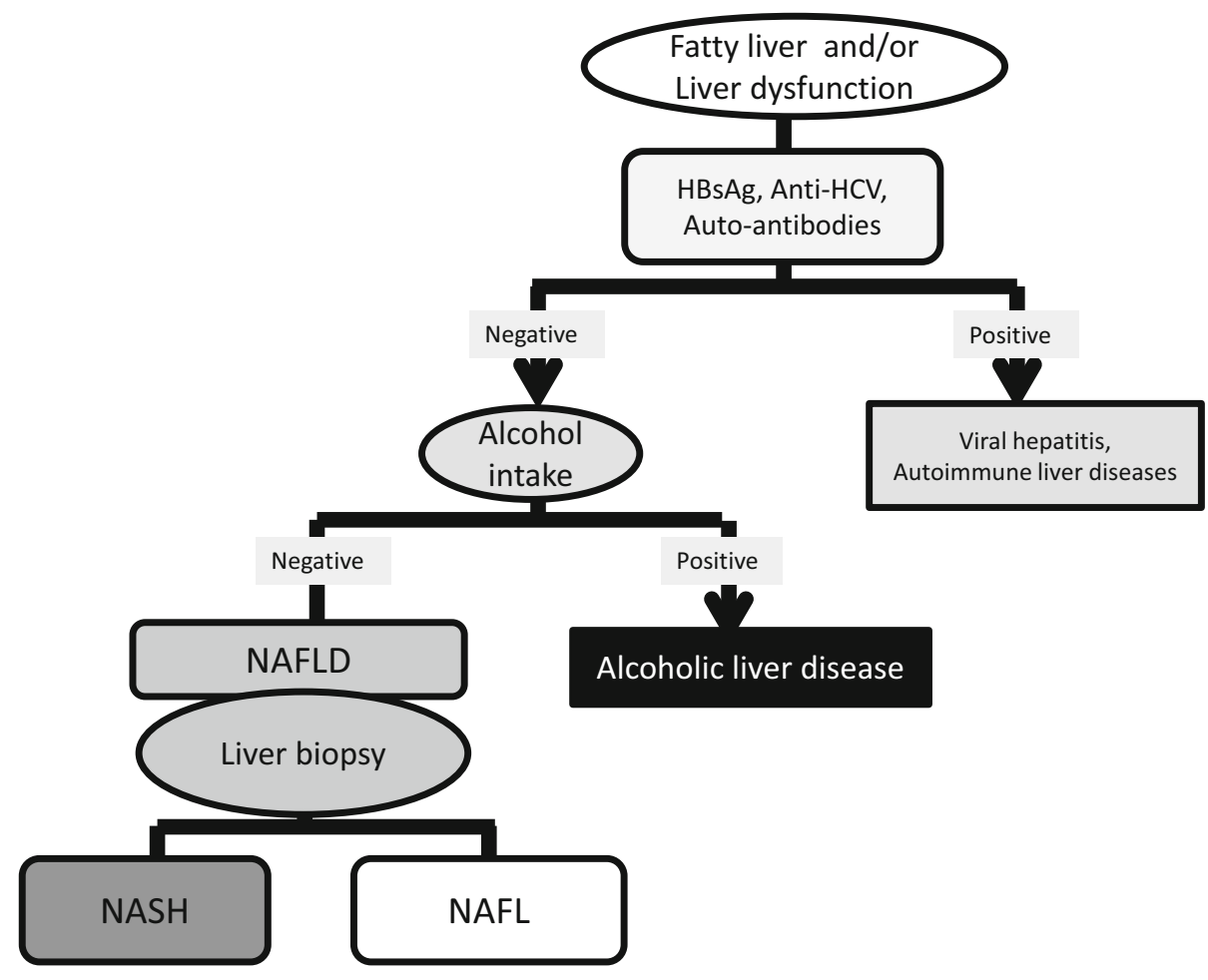

elastography or FibroScan, which measures liver stiffness noninvasively, has shown promising results for assessing the severity of liver fibrosis $[57,58]$.

\section{Pathological diagnosis of NASH}

NAFL (or non-NASH) is histologically defined as the presence of hepatic steatosis with no evidence of hepatocellular injury (no ballooning degeneration). NASH is defined as the presence of hepatic steatosis and inflammation together with hepatocyte injury (ballooning degeneration) $[10,11]$.

There are three important pathological classifications of NAFLD/NASH, which are the Matteoni classification [59], the Brunt classification [60], and the NAFLD activity score (NAS) [61]. In 1999, Matteoni et al. [59] described a classification system that served to distinguish between NASH and non-NASH. They divided 132 NAFLD patients into four categories: type 1, steatosis alone; type 2, steatosis with lobular inflammation only; type 3 , steatosis with hepatocellular ballooning, and type 4, type 3 plus either Mallory-Denk bodies or fibrosis. They confirmed the benign clinical course of patients with type 1 or 2 NAFLD and the progressive course of patients who had either type 3 or 4 NAFLD. As a result of these differences, these authors defined type 1 and 2 histological forms of NAFLD as "non-NASH" and type 3 and 4 as NASH. However, this classification did not include an assessment of the severity or pattern of NASH.
In the same year as the Matteoni classification system was published, Brunt et al. [60] proposed a semiquantitative grading and staging system for NASH. This classification was applicable only to NASH.

In 2005, the NASH Clinical Research Network Pathology Committee developed and validated NAS, which is a histological scoring system based on Brunt's classification, as a semiquantitative instrument by which to judge treatment responses or disease progression in clinical studies [61]. The NAS system addresses the full spectrum of NAFLD, and is applicable to both adult and pediatric NAFLD patients. The score is determined as the unweighted sum of the scores for steatosis (0-3), lobular inflammation (0-3), and ballooning degeneration (0-2). A score of greater than or equal to 5 correlated with the diagnosis of NASH made independently by an experienced pathologist without using the score. Likewise, scores less than 3 correlated with "non-NASH," and scores of 3 or 4 were regarded as borderline. With regard to fibrosis, stage 1 referred to perisinusoidal fibrosis in the perivenular area (stage 1A, delicate; and stage 1B, dense). Detection of portal fibrosis without perisinusoidal fibrosis was defined as stage $1 \mathrm{C}$. Stage 2 was characterized by perisinusoidal and portal/periportal fibrosis. Stage 3 was defined as bridging fibrosis, and stage 4 as cirrhosis. The authors emphasized that the definitive diagnosis of NASH did not always correlate with threshold values of NAS. Therefore, clinical pathologists should be encouraged not to use NAS as a categorical approach for the diagnosis of NASH. 


\section{Treatment of NAFLD/NASH}

NAFLD is usually associated with metabolic disturbance such as visceral obesity, insulin resistance, type 2 diabetes mellitus, and dyslipidemia, and these underlying conditions play crucial roles in its pathogenesis. In addition, Tilg and Moschen proposed the "parallel hit hypothesis" [31] in which many hits derived from gut and visceral adipose tissue may act in parallel to produce liver inflammation, subsequently leading to steatohepatitis. Therefore, it makes sense to treat not only liver disease itself but also these associated metabolic morbidities, and it is also essential to prevent the "hits" for the management of NAFLD/NASH. The effectiveness of several regimens for the treatment of NAFLD/NASH have been investigated, which include lifestyle intervention, surgical treatment, and pharmacotherapy. Among them, lifestyle modification, weight loss, and increased physical activity have been proved to be effective in many studies and represent the cornerstone of treatment. Given the important role of insulin resistance in the pathophysiology of NAFLD/NASH, several studies have attempted to investigate the effect of insulin-sensitizing agents, e.g., pioglitazone potentially improved serum ALT levels and histological features in NASH when administered over a short duration [62]. However, its safety regarding cardiovascular disease, congestive heart failure, and bladder cancer over the long term has not been fully assessed in any studies. Furthermore, many other medications including vitamin E, antioxidants, angiotensin II receptor blockers, statins, fibrates, ezetimibe, and hepatoprotective agents have also been investigated using animal models and in preliminary pilot studies. Vitamin E, an antioxidant, resulted in histological improvements, though the safety of long-term administration and the risk of excess usage still need to be discussed [63]. Bariatric surgery may be another option for treatment of NAFLD/NASH [64]. However, the benefit and harm, especially in patients with advanced liver disease, should be further evaluated.

The most important problem in these studies is that the majority of them were non-randomized, performed over relatively short durations, and they each had different endpoints and follow-up terms, which makes it difficult to construct firm evidence for the treatment of NAFLD/NASH.

Lifestyle-related interventions and weight loss surgery

\section{CQ: Does body weight reduction have a beneficial effect on NAFLD/NASH?}

- We recommend implementing body weight reduction through diet and exercise therapies for a period of 3-12 months to improve liver function and histology in NAFLD/NASH. (Evidence level A, strength 1.)
CQ: What dietary regimens are beneficial for improving NAFLD/NASH?

- Body weight reduction using a low-calorie diet improves liver function and fatty changes in the liver in patients with NAFLD. For improving NAFLD/NASH, we recommend prioritizing the optimization of energy intake and restricting lipids in terms of the proportions of nutrient intake. (Evidence level C, strength 2.)

\section{CQ: Is exercise beneficial for improving NAFLD/} NASH?

- Although the effects of exercise on liver histology have not been clarified, we recommend implementing exercise therapy because, even alone, it improves liver function and fatty changes in the liver in patients with NAFLD. (Evidence level B, strength 2.)

\section{CQ: Is bariatric surgery effective for improving NAFLD/NASH?}

- In patients with NAFLD/NASH complicated by severe obesity, weight loss surgery is effective for improving the fatty changes in the liver and inflammation associated with NASH. (Evidence level B, strength N/A.)

Lifestyle-related interventions such as diet and exercise therapies have been reported to improve serum transaminase levels as well as fatty changes in the liver as measured using US and MRI in patients with NAFLD. However, a meta-analysis of research papers published up to the year 2000 showed that many of the studies were non-randomized, and no histological improvements had been confirmed, and therefore no clear conclusions could be drawn as to the effectiveness of lifestyle-related interventions for NAFLD [65]. In a recent study, 31 patients with histologically confirmed NASH were randomly allocated to a group undergoing diet and exercise therapies for 48 weeks or a control group, and a mean decrease in body weight of $9.3 \%$ and improvements in NAS were reported in the former group. In addition, stratified analysis showed that a decrease in body weight of at least $7 \%$ was necessary for improving the NAS [66]. These findings indicate the effectiveness of body weight reduction for patients with obesity-related NAFLD/NASH.

In almost all reports of dietary interventions for patients with obesity-related NAFLD, a low-calorie diet is prescribed, and in terms of dietary contents, the proportions of energy intake from carbohydrates and lipids are often restricted to $50-60$ and $20-25 \%$, respectively. This proportion of intake from carbohydrates can also be considered appropriate from the perspective of ensuring compliance, but its adjustment is considered according to the disease state. In addition, it is known that low-fat diets decrease 
body weight, that combination of such diets with lowcalorie diets promotes weight loss, and that caloric restriction is more important than the proportions of carbohydrates and lipids for weight loss [67]. Exercise therapy is another useful lifestyle-related intervention for NAFLD/ NASH. Continuation of aerobic exercise in 30- to 60-min sessions held 3-4 times weekly for 4-12 weeks primarily in patients with NAFLD complicated by obesity has been shown to improve fatty changes in the liver, even without accompanying body weight reduction $[68,69]$. While these reports do not use histological changes as endpoints and show only short-term outcomes, fatty changes in the liver are thought to improve even with exercise therapy alone.

In cases of NASH complicated by severe obesity, weight loss surgery may be expected to be effective. A metaanalysis regarding weight loss surgery for 434 cases of NASH reported between 1998 and 2007 showed percentages of patients improving with regard to fatty changes in the liver $(91.6 \%)$, inflammation $(81.3 \%)$, and fibrosis $(65.5 \%)$ [64]. While weight loss surgery for severe obesity has also been performed in Japan, no reports exist regarding NAFLD/NASH, and this is an issue requiring future investigation.

Therefore, while body weight reduction using lifestylerelated interventions involving diet and exercise therapies or weight loss surgery is thought to be useful for patients with NAFLD/NASH, the intervention periods were relatively short, and investigation of effects over longer observation periods that also include hepatic fibrosis and hepatocarcinogenesis is desired in the future.

\section{Pharmacologic intervention}

Pharmacologic treatments for NAFLD/NASH are targeted at underlying metabolic syndrome-related diseases, such as obesity, type 2 diabetes mellitus, dyslipidemia, and hypertension, as well as liver dysfunction itself. To date, many drugs and supplements have been assessed for the treatment of NAFLD/NASH; however, fully proven medications for the diseases do not exist. On the other hand, the effects of several drugs and supplements on NAFLD/NASH have been evaluated by high quality clinical trials, such as randomized controlled trials (RCTs), and the evidence for the efficacy of some drugs and supplements is gradually accumulating.

\section{Thiazolidinediones}

\section{CQ: Are thiazolidinediones effective for patients with NAFLD/NASH?}

- Pioglitazone is recommended for NASH patients with insulin resistance. (Evidence level A, strength 2.)
Insulin resistance is considered to be related to pathogenesis and progression of NAFLD/NASH, and several studies have investigated the effect of thiazolidinediones, such as pioglitazone and rosiglitazone on aminotransferases and hepatic histology in patients with NASH; however, rosiglitazone is no longer marketed in Europe and its use is highly restricted in the USA because of increased risk of coronary diseases and, moreover, this drug is not sold in Japan. In regard to the effect of thiazolidinediones, there have been four RCTs, three for pioglitazone $[62,63,70]$ and one for rosiglitazone [4], and both drugs have had their efficacy against NASH confirmed by assessment of hepatic pathologic changes including steatosis, ballooning, and lobular inflammation. The largest and longest-term RCT was reported by Sanyal et al. [63]. They found that 96-week pioglitazone treatment induced improvement in serum biochemical indices and hepatic pathologic changes. In addition, there are two meta-analyses on thiazolidinediones $[71,72]$. Both reports showed the efficacy of pioglitazone against hepatic fibrosis. The long-term effect of pioglitazone remains to be determined, and pioglitazone's effects regarding hard endpoints, such as prevention of cirrhosis and long-term survival, have not been evaluated. Since pioglitazone has several side effects, including weight gain, heart failure, bone fracture, and bladder carcinoma, careful attention should be paid to long-term pioglitazone use.

\section{Vitamin E}

\section{CQ: Is vitamin $E$ effective for patients with NAFLD/ NASH?}

- Vitamin E improves hepatic biological and histological parameters in patients with NASH, and we recommend it for NASH patients. (Evidence level A, strength 2.)

Although much clinical, epidemiological, and basic research has aimed to clarify the mechanisms underlying development of NAFLD and NASH, the pathogenesis of NAFLD and NASH is not fully understood; however, oxidative stress is considered to be one of the main players in the development and progression of NAFLD/NASH [72-74]. Vitamin $E$ is known as a free radical scavenger, and a chainbreaking antioxidant in free radical reactions, such as lipid peroxidation. Vitamin $\mathrm{E}$ is thought to act on TGF- $\beta 1$, peroxisome proliferator-activated receptors, and apoptosisregulating genes. Two initial observational trials demonstrated the efficacy of vitamin E against NASH. Lavine et al. [73] gave vitamin E (400-1200 IU/day) to 11 obese NASH children for 4-10 months and found normalization of serum aminotransferase; however, they did not evaluate histological efficacy. Hasegawa et al. [74] chose 12 adult patients with biopsy-proven NASH and gave vitamin E (300 IU/day) for 12 months, and then the improvements in hepatic 
inflammation and fibrosis as well as serum aminotransferase were observed. Since then, several open label studies and RCTs on vitamin E and NASH have been conducted. Comparison between these trials is difficult because of the varying criteria for entry into the study, different doses of vitamin E, and unclear formulations of vitamin E used which could affect its bioavailability. Two large RCTs, the PIVEN study and the TONIC study, have were recently presented [63, 75]. In the largest clinical trial reported (PIVEN), vitamin E was administered orally at a dose of 800 IU/day for 96 weeks, and it was confirmed that vitamin $\mathrm{E}$ has beneficial efficacy against NASH by improving serum biochemical indices and hepatic pathologic changes [63]. The long-term effect of vitamin E remains to be determined and its effects regarding hard endpoints, such as prevention of cirrhosis and long-term survival, have not been evaluated. Since some meta-analyses have reported an increase in all-cause mortality with high dose vitamin E, careful attention should be paid to the largedose and long-term use of vitamin $\mathrm{E}$.

\section{Metformin}

\section{CQ: Are biguanides effective for patients with NAFLD/ NASH?}

- Metformin has no significant effect on liver histology and we do not recommend it as a specific treatment for liver disease in patients with NASH. (Evidence level B, strength 2.)

Several studies investigated the effect of metformin on NASH. Four RCTs have been reported. The first RCT by Uygun et al. [76] showed that metformin induced only modest improvement in serum biochemical indices, but not hepatic histology. Nar and Gedik [77] reported no significant efficacy of metformin against NAFLD as assessed by US in patients with concurrent NAFLD and type 2 diabetes mellitus. Haukeland et al. [78] reported a similar lack of efficacy in a larger RCT. Other studies also failed to show any major benefit of metformin on serum biochemical indices aside from improvement of arterial resistance [79]. Four recent meta-analyses were unable to conclude that metformin is beneficial for NAFLD/NASH [80-83]. On the basis of these studies, metformin is not recommended for the treatment of NAFLD/NASH.

\section{Ursodeoxycholic acid (UDCA)}

CQ: Are conventional doses of ursodeoxycholic acid effective for patients with NAFLD/NASH?

- We do not recommend UDCA at conventional dose levels for the treatment of NAFLD or NASH. (Evidence level B, strength 2.)
UDCA is used in a wide variety of liver diseases including primary biliary cirrhosis and viral chronic hepatitis because of its hepatic cytoprotective effect. Several studies investigated UDCA with conventional and high doses to improve serum biochemical indices and hepatic histology in patients with NAFLD/NASH. An RCT for UDCA at conventional doses for NASH was reported in 2004, and conventional doses of UDCA did not show any beneficial effects on serum biochemical indices and hepatic histology in patients with NASH [84]. An RCT for high doses of UDCA on NASH was published in 2010, but did not show overall improvement in serum biochemical indices and hepatic histology aside from modest improvement in serum $\gamma$-GTP levels [85]. A meta-analysis also revealed that UDCA induced only modest improvement in serum ALT, but overall improvement was not observed [86]. In 2011, an RCT for high dose UDCA treatment of NASH was published, and the results were very negative [87]. On the basis of these studies, conventional dosing of UDCA is not recommended for the treatment of NAFLD/NASH.

\section{Lipid-lowering agents}

\section{CQ: Are HMG-CoA reductase inhibitors effective for patients with NAFLD/NASH?}

- HMG-CoA reductase inhibitors (statins) are recommended for NAFLD/NASH patients with hypercholesterolemia. (Evidence level B, strength 2.)

\section{CQ: Is ezetimibe effective for hypercholesteremic patients with NAFLD/NASH?}

- Ezetimibe is recommended for NAFLD/NASH patients with hypercholesterolemia. (Evidence level C, strength 2.)

Two types of lipid-lowering agents have been tried for the treatment of NAFLD/NASH, namely HMG-CoA reductase inhibitors (statins) and ezetimibe. Statins are inhibitors of hepatic cholesterol synthesis and widely used for patients with hypercholesterolemia.

Statins are also known to act as PPAR- $\gamma$-like components, and several clinical trials have attempted to determine the efficacy of statins against NAFLD/NASH. Three RCTs were published in regard to statins and NAFLD/ NASH. Samy et al. [88] showed the beneficial efficacy of atorvastatin, but unfortunately they did not estimate hepatic histology. Foster et al. [89] conducted an RCT using atorvastatin plus antioxidants in NAFLD patients, and thus in their study it is not possible to evaluate the effect of statins alone. Likewise, Athyros et al. [90] reported an RCT for statins on NAFLD/NASH, but administered statins together with hypertensive drugs and insulin sensitizers, which may have beneficial effects on NAFLD/NASH. Only 
two pilot studies reported the efficacy of monotherapy with statins against NASH by evaluating the change in hepatic histology, but these studies both involved very small populations [91, 92].

Ezetimibe is an antagonist of Niemann-Pick C1-like protein, which is a key player in cholesterol absorption from the small intestine. Ezetimibe was released onto the market in the USA in 2002. Three Japanese groups reported pilot studies for estimating the effect of ezetimibe and they showed the clinical efficacy of ezetimibe against NAFLD/NASH by evaluating serum biochemical indices and hepatic histology [93-95].

\section{Angiotensin II receptor antagonist (ARB)}

CQ: Are angiotensin II receptor antagonists effective for hypertensive patients with NAFLD/NASH?

- We recommend angiotensin II receptor antagonists (ARB) for NASH patients with hypertension. (Evidence level C, strength 2.)

ARBs are widely used for patients with hypertension, and are known to inhibit fibroblast activity, resulting in inhibition of tissue fibrosis in several organs. In the liver, stellate cells have angiotensin II type 1 receptors on their surface, and angiotensin binds to these receptors and activates stellate cells. In 2004, a clinical pilot study revealed the beneficial efficacy of losartan, one of the ARBs, against NASH by evaluating serum biochemical indices and hepatic histology [96]. Moreover, the effect of ARB on NASH was shown to be through the inhibition of activated hepatic stellate cells in NASH patients [97]. After this pilot study, many animal and in vitro studies confirmed the beneficial efficacy of ARBs against NASH, but clinical studies were very limited. Only one RCT was published in 2009 [98]; however, this RCT was conducted with two treatment groups, and each group of patients was given a different ARB. This RCT reported that both telmisartan and valsartan have beneficial efficacy against NASH, but there was no placebo treatment group [98]. Thus, it is difficult to assess whether the beneficial efficacy against NASH is specific to ARBs or whether other antihypertensive agents might also have the same effect on NASH.

\section{Other drugs}

\section{CQ: Are there any other drugs effective for patients with NAFLD/NASH?}

- Pentoxifylline is recommended for NASH patients; however, pentoxifylline is not commercially available in Japan. (Evidence level A, strength N/A.)
- Betaine has no significant effect on liver function, and we do not recommend it as a specific treatment for liver disease in patients with NASH. (Evidence level B, strength 1.)

In the pathogenesis of NAFLD/NASH, oxidative stress and inflammatory cytokines play central roles, and pentoxifylline is considered to have both antioxidant and anti-TNF- $\alpha$ effects. Thus, several studies tried to test the efficacy of pentoxifylline against NAFLD/NASH. Two RCTs and two meta-analyses were conducted, and these studies found that pentoxifylline improved serum biochemical indices and hepatic histological change [86, 99-101]; however, this drug is no longer marketed in Japan, because its initial efficacy for treating aftereffects of brain stroke was reevaluated and found to be insufficient.

Betaine is also considered to be an antioxidant, and is widely used for NAFLD/NASH mainly in the form of supplements. Two RCTs were conducted to confirm the effect of betaine on NAFLD/NASH; however, one involved a combination therapy with other drugs, and in the other, betaine monotherapy did not show beneficial efficacy against NAFLD/NASH [102].

\section{Liver transplantation}

\section{CQ: Is liver transplantation effective for advanced} NASH patients with hepatic failure?

- We recommend liver transplantation for advanced NASH patients with hepatic failure, because overall survival after transplantation is approximately identical with patients who receive liver transplants for hepatic failure caused by other liver diseases. (Evidence level B, strength 2.)

Several cohort studies were conducted to confirm the usefulness of liver transplantation for end-stage NASH cirrhotic patients. An RCT for liver transplantation cannot be conducted for ethical reasons. Overall results of reported cohort studies indicated that survival after liver transplantation for NASH patients was approximately the same as for liver transplant patients with other causes of hepatic failure [103-108].

\section{Iron reduction therapy}

\section{CQ: Is phlebotomy effective for improving NAFLD/} NASH?

- We do not recommend iron reduction therapy for NAFLD/NASH at present. (Evidence level C, strength 2.) 
Although several therapeutic trials including diet, antioxidants, and approaches that improve insulin resistance have been tried for NAFLD/NASH, no optimal therapy has been established. It has been reported that elevated serum iron levels and increased hepatic tissue iron deposit are relatively common in NAFLD/NASH. More importantly, elevated iron levels were associated with hepatic inflammation, fibrosis progression, and insulin resistance in patients with NAFLD/NASH [109], suggesting iron reduction therapy as an attractive and potentially promising therapeutic modality for NAFLD/NASH. Facchini et al. [110] have shown improvement in liver enzyme levels in 17 NAFLD patients with impaired glucose tolerance undergoing serial phlebotomy. According to Fargion et al. [111], in 42 NAFLD patients, serum transaminase levels were significantly decreased after a 4-month hypocaloric diet, and a further reduction was observed after phlebotomies. Sumida et al. [112] also reported that phlebotomy decreased serum transaminase activities in Japanese patients with biopsy-proven NASH. Riquelme et al. [113] reported that a 52-year-old nonobese woman with biopsy-proven NASH obtained not only improvement in transaminase activities but also complete resolution of fatty infiltration and inflammatory changes in liver histology after iron depletion therapy (long-term phlebotomy with a low-iron diet). According to a case-control study [114], iron depletion produced a significantly larger decrease in ALT activities compared with nutritional counseling alone, independent of changes in BMI and the presence of the metabolic syndrome. Since the sample sizes of these studies were relatively small and the durations were short, further validation studies are required.

\section{Principles of therapy for NAFLD/NASH}

Therapy for NAFLD depends on the histological findings (Fig. 1). If biopsy histology shows the patient to have simple hepatic steatosis without steatohepatitis or liver fibrosis, lifestyle modification, weight loss, and increased physical activity are recommended, though bariatric surgery may be another option if the patient is severely obese. In cases with NASH, the treatment should vary according to the underlying disease. If the patient does not have associated disease, simple weight loss by lifestyle modification and increased physical activity should be sufficient, as in cases with simple steatosis. If the patient has another underlying metabolic disturbance such as insulin resistance, type 2 diabetes mellitus, dyslipidemia, and hypertension, therapies for these associated diseases will be necessary in addition to weight loss.
Future prospects

Recently, several lines of evidence have shown that NAFLD/NASH affects both life-span and quality of life. According to several population-based cohort studies, individuals with NAFLD/NASH have a shorter life expectancy than the general population [115-122]. Some cohort studies have shown that the prevalence of NAFLD/ $\mathrm{NASH}$ is a risk factor independent of cardiovascular disease even after correction for such factors as metabolic disease and age [123, 124], and a meta-analysis demonstrated that NAFLD almost doubles the risk of cardiovascular events compared with the normal population (odds ratio $=2.05$ ) [125]. The liver disease-related mortality rate for NASH is worse than that for NAFL and the general population [115-117, 125]. In a cohort study involving biopsy-based diagnoses distinguishing 173 non-NASH from 72 NASH patients, the hazard ratio for liver-related death in NASH patients compared with non-NASH was as high as 13.9 [117].

The incidence of HCC in patients with NASH appears to be lower than in patients with chronic hepatitis $\mathrm{C}$, although the exact rate for NAFLD overall remains unknown. A cohort study has shown that HCC incidence in NASHbased cirrhosis is $2.6 \%$ /year whereas HCC incidence in chronic hepatitis C-based cirrhosis is $4.0 \%$ year [126]. Characteristics of patients with NASH-based HCC according to case reports are advanced age and liver cirrhosis with complications of metabolic syndrome [127]. Type 2 diabetes mellitus in particular contributes to liver carcinogenesis in NASH. In a large controlled trial involving 173,643 patients with type 2 diabetes and 650,620 people without diabetes in the USA, the hazard ratio for liver carcinogenesis among diabetic patients was 2.16 [128]. The prognosis for NASH-based HCC is equal to or better than hepatitis C-based HCC [129]. Preventing the progression of NAFLD promises to improve not only liverrelated complications but also extra-liver complications. However, we have yet to achieve strong evidence for specific treatments for NAFLD/NASH as described above. Obviously, further studies (RCTs) with concurrent endpoints and longer follow-up periods, and assessment of both efficacy and safety over the long term are necessary for each treatment in the near future. In addition, new modalities/biomarkers are needed that can monitor the progression/regression of the disease as well as the effect of the therapy.

In considering how to develop the present guideline, the priorities were to establish a clinically useful and internationally accepted guideline. NAFLD/NASH are relatively new disease concepts, so it was important to clearly define the diseases and set a goal for their treatment. Next, we 
assessed currently available evidence therapy by therapy and presented the process of treatments as a decision tree to avoid exclusive treatments based on uncertain evidence. The outcome of our analysis is that vitamin E and thiazolidinedione derivatives are the most evidence-based therapeutic options, although the clinical evidence for long-term efficacy and safety is limited. Statins/ARBs are also recommended therapies depending on the complications. Elucidating the pathogenesis of NAFLD/NASH and developing therapies are now worldwide issues, and so Japanese medical studies of NAFLD/NASH need to advance together with those in other countries. For that purpose, this guideline was prepared by globally searching for relevant evidence worldwide without regard to ethnic characteristics, and this evidence was summarized from a Japanese perspective. Of course, ethnic differences are correlated with susceptibility to metabolic syndrome-related diseases including NAFLD/NASH [130], possibly as a result of genomic polymorphism in genes for PPAR- $\gamma$ [131], insulin receptor signaling [132], or lipid metabolism [133]. Therefore, it is also important to develop Japanesebased clinical research work and interpret other countries' evidence in light of ethnic considerations.

Surely the present guideline should be updated in the future in response to new evidence, because the study of NAFLD/NASH is a constantly advancing field. Actually, some promising clinical trials for treatment of NAFLD/ NASH by molecularly targeted therapies are ongoing. The present guideline not only summarizes the current clinical state of NAFLD/NASH but also indicates future directions for study.

Acknowledgments This article was supported by a Grant-in-Aid from the Japanese Society of Gastroenterology. The authors thank R. F. Whittier (Mitsui Plant Biotechnology Research Institute) for critical reading of the manuscript and thoughtful discussions and Kazuyoshi Kon (Juntendo University) for great assistance with manuscript preparation. The authors also express special appreciation to Shunhei Yamashina, Reiko Yaginuma, Kyoko Fukuhara, Akira Uchiyama, Tomonori Aoyama (Juntendo University), Seiichi Mawatari, Kohei Oda, Kazuaki Tabu (Kagoshima University), Takeshi Okanoue (Saiseikai Suita Hospital), Naoki Fujita, Motoo Iwasa, Kazushi Sugimoto (Mie University), Takuya Miyagi, Yoshihiro Kamada (Osaka University), Takaomi Kessoku, Wataru Tomeno, Kento Imajo, Masato Yoneda, Yuji Ogawa (Yokohama City University), Haruhisa Nakao, Kiyoaki Ito, Ken Sato and Yukiomi Nakade (Aichi Medical University).

Conflict of interest Any financial relationship with enterprises, businesses, or academic institutions in the subject matter or materials discussed in the manuscript are listed as follows: (1) those from which the authors, the spouse, partner, or immediate relatives of authors have received individually any income, honoraria, or any other types of remuneration-Astellas Pharma Inc., Ishiyaku Pub, Inc., Eisai Co., MSD K. K., Otsuka Pharmaceutical Co., Ltd., Daiichi Sankyo Company, Limited, Takeda Pharmaceutical Company Limited., Mitsubishi Tanabe Pharma Corporation, Chugai Pharmaceutical Co., Ltd., Nippon Shinyaku Co., Ltd., Bristol-Myers Squibb Company,
Lundbeck Japan K. K.; and (2) those from which the academic institutions of the authors received support (commercial/academic cooperation)-Ajinomoto Pharmaceuticals Co., Ltd., Astellas Pharma Inc., AstraZeneca K. K., Eisai Co., Ltd., MSD K. K., Otsuka Pharmaceutical Co., Ltd., Kaneka Corporation, Kan Research Institute, Inc. Kyorin Pharmaceutical Co., Ltd., Suntory Holdings Limited., Shionogi \& Co., Ltd., Jimro Co. Ltd., Daiichi Sankyo Company, Limited, Sumitomo Dainippon Pharma Co., Ltd., Taiho Pharmaceutical Co., Ltd., Takara Bio Inc., Takeda Pharmaceutical Company Limited., Mitsubishi Tanabe Pharma Corporation, Chugai Pharmaceutical Co., Ltd., Toray Industries, Inc., Boehringer Ingelheim Japan, Inc., Bayer Yakuhin, Ltd., Bristol-Myers Squibb Company, Minophagen Pharmaceutical Co., Ltd., Mochida Pharmaceutical Co., Ltd., Yakult Honsha Co., Ltd., Janssen Pharmaceutical K.K., Rohto Pharmaceutical Co., Ltd.

\section{Appendix}

Members of the Working Committee who created and evaluated the JSGE "Evidence-based clinical practice guidelines for nonalcoholic fatty liver disease/nonalcoholic steatohepatitis" are listed below.

\section{Director Responsible}

Hirohito Tsubouchi (Digestive and Lifestyle Diseases, Department of Human and Environmental Sciences, Kagoshima University Graduate School of Medical and Dental Sciences)

\section{Executive Committee}

Chair: Sumio Watanabe (Department of Gastroenterology, Juntendo University, School of Medicine)

Vice Chair: Etsuko Hashimoto (Department of Medicine and Gastroenterology, Tokyo Women's Medical University)

Members: Kenichi Ikejima (Department of Gastroenterology, Juntendo University, School of Medicine), Hirofumi Uto (Digestive and Lifestyle Diseases, Department of Human and Environmental Sciences, Kagoshima University Graduate School of Medical and Dental Sciences), Masafumi Ono (Department of Gastroenterology and Hepatology, Kochi Medical School), Yoshio Sumida (Department of Gastroenterology and Hepatology, Kyoto Prefectural University of Medicine), Masataka Seike (Department of Gastroenterology, Faculty of Medicine, Oita University), Yoshiyuki Takei (Department of Gastroenterology and Hepatology, Mie University Graduate School of Medicine), Tetsuo Takehara (Department of Gastroenterology and Hepatology, Osaka University Graduate School of Medicine), Katsutoshi Tokushige (Department of Medicine and Gastroenterology, Tokyo Women's Medical University), Atsushi Nakajima (Division of Gastroenterology Graduate School of Medicine Yokohama City University), Masashi Yoneda (Division of Gastroenterology, Department of Internal Medicine, Aichi Medical University) 


\section{Evaluation Committee}

Chair: Toshiji Saibara (Department of Gastroenterology and Hepatology, Kochi Medical School)

Vice-Chair: Goshi Shiota (Department of Genetic Medicine and Regenerative Therapeutics, Tottori University Faculty of Medicine)

Members: Isao Sakaida (Department of Gastroenterology and Hepatology, Yamaguchi University Graduate School of Medicine), Makoto Nakamuta (Department of Gastroenterology, Kyushu Medical Center, National Hospital Organization), Toshihiko Mizuta (Department of Internal Medicine, Saga Medical School)

\section{The Japanese Society of Gastroenterology}

President, Tooru Shimosegawa (Division of Gastroenterology, Tohoku University Graduate School of Medicine); Former President, Kentaro Sugano (Jichi Medical University).

\section{References}

1. Komeda T. Obesity and NASH in Japan. Hepatol Res. 2005;33:83-6.

2. Jimba S, Nakagami T, Takahashi M, et al. Prevalence of nonalcoholic fatty liver disease and its association with impaired glucose metabolism in Japanese adults. Diabet Med. 2005;22:1141-5.

3. Hamaguchi M, Kojima T, Takeda N, et al. The metabolic syndrome as a predictor of nonalcoholic fatty liver disease. Ann Intern Med. 2005;143:722-8.

4. Vernon G, Baranova A, Younossi ZM. Systematic review: the epidemiology and natural history of non-alcoholic fatty liver disease and non-alcoholic steatohepatitis in adults. Aliment Pharmacol Ther. 2011;34:274-85.

5. Chen $\mathrm{CH}$, Huang $\mathrm{MH}$, Yang JC, et al. Prevalence and risk factors of nonalcoholic fatty liver disease in an adult population of Taiwan: metabolic significance of nonalcoholic fatty liver disease in nonobese adults. $\mathrm{J}$ Clin Gastroenterol. 2006;40:745-52.

6. Kojima S, Watanabe $\mathrm{N}$, Numata $\mathrm{M}$, et al. Increase in the prevalence of fatty liver in Japan over the past 12 years: analysis of clinical background. J Gastroenterol. 2003;38:954-61.

7. Sanyal AJ. AGA technical review on nonalcoholic fatty liver disease. Gastroenterology. 2002;123:1705-25.

8. Chan HL, de Silva HJ, Leung NW, et al. How should we manage patients with non-alcoholic fatty liver disease in 2007 ? J Gastroenterol Hepatol. 2007;22:801-8.

9. Okanoue T, Saibara T, Ono M, et al. JSH Consensus Kobe 2009; diagnosis and treatment of NASH. Kanzo. 2009;50:741-7.

10. Ratziu V, Bellentani S, Cortez-Pinto H, et al. A position statement on NAFLD/NASH based on the EASL 2009 special conference. J Hepatol. 2010;53:372-84.

11. Chalasani N, Younossi Z, Lavine JE, et al. The diagnosis and management of non-alcoholic fatty liver disease: practice Guideline by the American Association for the Study of Liver Diseases, American College of Gastroenterology, and the American Gastroenterological Association. Hepatology. 2012;55:2005-23.

12. Bellentani S, Saccoccio G, Costa G, et al. Drinking habits as cofactors of risk for alcohol induced liver damage. The Dionysos Study Group. Gut. 1997;41:845-50.
13. Bellentani S, Saccoccio G, Masutti F, et al. Prevalence of and risk factors for hepatic steatosis in Northern Italy. Ann Intern Med. 2000;132:112-7.

14. Eguchi Y, Hyogo H, Ono M, et al. Prevalence and associated metabolic factors of nonalcoholic fatty liver disease in the general population from 2009 to 2010 in Japan: a multicenter large retrospective study. J Gastroenterol. 2012;47:586-95.

15. Williams CD, Stengel J, Asike MI, et al. Prevalence of nonalcoholic fatty liver disease and nonalcoholic steatohepatitis among a largely middle-aged population utilizing ultrasound and liver biopsy: a prospective study. Gastroenterology. 2011;140:124-31.

16. Radu C, Grigorescu M, Crisan D, et al. Prevalence and associated risk factors of non-alcoholic fatty liver disease in hospitalized patients. J Gastrointestin Liver Dis. 2008;17:255-60.

17. Hou XH, Zhu YX, Lu HJ, et al. Non-alcoholic fatty liver disease's prevalence and impact on alanine aminotransferase associated with metabolic syndrome in the Chinese. J Gastroenterol Hepatol. 2011;26:722-30.

18. Angulo P. GI epidemiology: nonalcoholic fatty liver disease. Aliment Pharmacol Ther. 2007;25:883-9.

19. Yatsuji S, Hashimoto E, Tobari M, et al. Influence of age and gender in Japanese patients with non-alcoholic steatohepatitis. Hepatol Res. 2007;37:1034-43.

20. Browning JD, Szczepaniak LS, Dobbins R, et al. Prevalence of hepatic steatosis in an urban population in the US: impact of ethnicity. Hepatology. 2004;40:1387-95.

21. Hashimoto E, Tokushige K. Prevalence, gender, ethnic variations, and prognosis of NASH. J Gastroenterol. 2011;46(Suppl 1):63-9.

22. Caldwell SH, Oelsner DH, Iezzoni JC, et al. Cryptogenic cirrhosis: clinical characterization and risk factors for underlying disease. Hepatology. 1999;29:664-9.

23. Yoshioka Y, Hashimoto E, Yatsuji S, et al. Nonalcoholic steatohepatitis: cirrhosis, hepatocellular carcinoma, and burntout NASH. J Gastroenterol. 2004;39:1215-8.

24. Michitaka K, Nishiguchi S, Aoyagi Y, et al. Etiology of liver cirrhosis in Japan: a nationwide survey. J Gastroenterol. 2010;45:86-94.

25. Tokushige K, Hashimoto E, Horie Y, et al. Hepatocellular carcinoma in Japanese patients with nonalcoholic fatty liver disease, alcoholic liver disease, and chronic liver disease of unknown etiology: report of the nationwide survey. J Gastroenterol. 2011;46:1230-7.

26. Mencin AA, Lavine JE. Nonalcoholic fatty liver disease in children. Curr Opin Clin Nutr Metab Care. 2011;14:151-7.

27. Loomba R, Sirlin CB, Schwimmer JB, et al. Advances in pediatric nonalcoholic fatty liver disease. Hepatology. 2009;50:1282-93.

28. Nobili V, Alisi A, Panera N, et al. Low birth weight and catchup-growth associated with metabolic syndrome: a 10 year systematic review. Pediatr Endocrinol Rev. 2008;6:241-7.

29. Nobili V, Marcellini M, Marchesini G, et al. Intrauterine growth retardation, insulin resistance, and nonalcoholic fatty liver disease in children. Diabetes Care. 2007;30:2638-40.

30. Day CP, James OF. Steatohepatitis: a tale of two "hits"? Gastroenterology. 1998;114:842-5.

31. Tilg H, Moschen AR. Evolution of inflammation in nonalcoholic fatty liver disease: the multiple parallel hits hypothesis. Hepatology. 2010;52:1836-46.

32. Tokushige K, Yatsuji S, Hashimoto E, et al. Familial aggregation in patients with non-alcoholic steatohepatitis. Intern Med. 2008;47:405-10.

33. Romeo S, Kozlitina J, Xing $\mathrm{C}$, et al. Genetic variation in PNPLA3 confers susceptibility to nonalcoholic fatty liver disease. Nat Genet. 2008;40:1461-5. 
34. Kawaguchi T, Sumida Y, Umemura A, et al. Genetic polymorphisms of the human PNPLA3 gene are strongly associated with severity of non-alcoholic fatty liver disease in Japanese. PLoS One. 2012;7:e38322.

35. Hotta K, Yoneda M, Hyogo H, et al. Association of the rs738409 polymorphism in PNPLA3 with liver damage and the development of nonalcoholic fatty liver disease. BMC Med Genet. 2010;11:172.

36. Tokushige K, Takakura M, Tsuchiya-Matsushita N, et al. Influence of TNF gene polymorphisms in Japanese patients with NASH and simple steatosis. J Hepatol. 2007;46:1104-10.

37. Farrell GC. Drugs and steatohepatitis. Semin Liver Dis. 2002;22:185-94.

38. Tanaka N, Horiuchi A, Yokoyama T, et al. Clinical characteristics of de novo nonalcoholic fatty liver disease following pancreaticoduodenectomy. J Gastroenterol. 2011;46:758-68.

39. Loria P, Carulli L, Bertolotti M, et al. Endocrine and liver interaction: the role of endocrine pathways in NASH. Nat Rev Gastroenterol Hepatol. 2009;6:236-47.

40. Targher G, Montagnana M, Salvagno G, et al. Association between serum TSH, free T4 and serum liver enzyme activities in a large cohort of unselected outpatients. Clin Endocrinol (Oxf). 2008;68:481-4.

41. Adams LA, Feldstein A, Lindor KD, et al. Nonalcoholic fatty liver disease among patients with hypothalamic and pituitary dysfunction. Hepatology. 2004;39:909-14.

42. Baranova A, Tran TP, Birerdinc A, et al. Systematic review: association of polycystic ovary syndrome with metabolic syndrome and non-alcoholic fatty liver disease. Aliment Pharmacol Ther. 2011;33:801-14.

43. Takahashi Y, Iida K, Takahashi K, et al. Growth hormone reverses nonalcoholic steatohepatitis in a patient with adult growth hormone deficiency. Gastroenterology. 2007;132:938-43.

44. Mofrad P, Contos MJ, Haque M, et al. Clinical and histologic spectrum of nonalcoholic fatty liver disease associated with normal ALT values. Hepatology. 2003;37:1286-92.

45. Amarapurkar DN, Patel ND. Clinical spectrum and natural history of non-alcoholic steatohepatitis with normal alanine aminotransferase values. Trop Gastroenterol. 2004;25:130-4.

46. Kaneda H, Hashimoto E, Yatsuji S, et al. Hyaluronic acid levels can predict severe fibrosis and platelet counts can predict cirrhosis in patients with nonalcoholic fatty liver disease. J Gastroenterol Hepatol. 2006;21:1459-65.

47. Angulo P, Keach JC, Batts KP, et al. Independent predictors of liver fibrosis in patients with nonalcoholic steatohepatitis. Hepatology. 1999;30:1356-62.

48. Guha IN, Parkes J, Roderick PR, et al. Non-invasive markers associated with liver fibrosis in non-alcoholic fatty liver disease. Gut. 2006;55:1650-60.

49. Harrison SA, Kadakia S, Lang KA, et al. Nonalcoholic steatohepatitis: what we know in the new millennium. Am J Gastroenterol. 2002;97:2714-24.

50. Wieckowska A, Zein NN, Yerian LM, et al. In vivo assessment of liver cell apoptosis as a novel biomarker of disease severity in nonalcoholic fatty liver disease. Hepatology. 2006;44:27-33.

51. Sumida Y, Yoneda M, Hyogo H, et al. A simple clinical scoring system using ferritin, fasting insulin, and type IV collagen $7 \mathrm{~S}$ for predicting steatohepatitis in nonalcoholic fatty liver disease. J Gastroenterol. 2011;46:257-68.

52. Angulo P, Hui JM, Marchesini G, et al. The NAFLD fibrosis score: a noninvasive system that identifies liver fibrosis in patients with NAFLD. Hepatology. 2007;45:846-54.

53. Guha IN, Parkes J, Roderick P, et al. Noninvasive markers of fibrosis in nonalcoholic fatty liver disease: validating the European liver fibrosis panel and exploring simple markers. Hepatology. 2008;47:455-60.
54. Hernaez R, Lazo M, Bonekamp S, et al. Diagnostic accuracy and reliability of ultrasonography for the detection of fatty liver: a meta-analysis. Hepatology. 2011;54:1082-90.

55. Saadeh S, Younossi ZM, Remer EM, et al. The utility of radiological imaging in nonalcoholic fatty liver disease. Gastroenterology. 2002;123:745-50.

56. Roldan-Valadez E, Favila R, Martinez-Lopez M, et al. Imaging techniques for assessing hepatic fat content in nonalcoholic fatty liver disease. Ann Hepatol. 2008;7:212-20.

57. Yoneda M, Yoneda M, Mawatari H, et al. Noninvasive assessment of liver fibrosis by measurement of stiffness in patients with nonalcoholic fatty liver disease (NAFLD). Dig Liver Dis. 2008;40:371-8.

58. Obara N, Ueno Y, Fukushima K, et al. Transient elastography for measurement of liver stiffness measurement can detect early significant hepatic fibrosis in Japanese patients with viral and nonviral liver diseases. J Gastroenterol. 2008;43:720-8.

59. Matteoni CA, Younossi ZM, Gramlich T, et al. Nonalcoholic fatty liver disease: a spectrum of clinical and pathological severity. Gastroenterology. 1999;116:1413-9.

60. Brunt EM, Janney CG, Di Bisceglie AM, et al. Nonalcoholic steatohepatitis: a proposal for grading and staging the histological lesions. Am J Gastroenterol. 1999;94:2467-74.

61. Kleiner DE, Brunt EM, Van Natta M, et al. Design and validation of a histological scoring system for nonalcoholic fatty liver disease. Hepatology. 2005;41:1313-21.

62. Belfort R, Harrison SA, Brown K, et al. A placebo-controlled trial of pioglitazone in subjects with nonalcoholic steatohepatitis. N Engl J Med. 2006;355:2297-307.

63. Sanyal AJ, Chalasani N, Kowdley KV, et al. Pioglitazone, vitamin E, or placebo for nonalcoholic steatohepatitis. N Engl J Med. 2010;362:1675-85.

64. Mummadi RR, Kasturi KS, Chennareddygari S, et al. Effect of bariatric surgery on nonalcoholic fatty liver disease: systematic review and meta-analysis. Clin Gastroenterol Hepatol. 2008;6:1396-402.

65. Wang RT, Koretz RL, Yee HF Jr. Is weight reduction an effective therapy for nonalcoholic fatty liver? A systematic review. Am J Med. 2003;115:554-9.

66. Promrat K, Kleiner DE, Niemeier HM, et al. Randomized controlled trial testing the effects of weight loss on nonalcoholic steatohepatitis. Hepatology. 2010;51:121-9.

67. Sacks FM, Bray GA, Carey VJ, et al. Comparison of weight-loss diets with different compositions of fat, protein, and carbohydrates. N Engl J Med. 2009;360:859-73.

68. van der Heijden GJ, Wang ZJ, Chu ZD, et al. A 12-week aerobic exercise program reduces hepatic fat accumulation and insulin resistance in obese, Hispanic adolescents. Obesity (Silver Spring). 2010;18:384-90.

69. Johnson NA, Sachinwalla T, Walton DW, et al. Aerobic exercise training reduces hepatic and visceral lipids in obese individuals without weight loss. Hepatology. 2009;50:1105-12.

70. Aithal GP, Thomas JA, Kaye PV, et al. Randomized, placebocontrolled trial of pioglitazone in nondiabetic subjects with nonalcoholic steatohepatitis. Gastroenterology. 2008;135:1176-84.

71. Mahady SE, Webster AC, Walker S, et al. The role of thiazolidinediones in non-alcoholic steatohepatitis-a systematic review and meta analysis. J Hepatol. 2011;55:1383-90.

72. Boettcher E, Csako G, Pucino F, et al. Meta-analysis: pioglitazone improves liver histology and fibrosis in patients with nonalcoholic steatohepatitis. Aliment Pharmacol Ther. 2012;35:66-75.

73. Lavine JE. Vitamin E treatment of nonalcoholic steatohepatitis in children: a pilot study. J Pediatr. 2000;136:734-8.

74. Hasegawa T, Yoneda M, Nakamura K, et al. Plasma transforming growth factor-betal level and efficacy of alpha- 
tocopherol in patients with non-alcoholic steatohepatitis: a pilot study. Aliment Pharmacol Ther. 2001;15:1667-72.

75. Lavine JE, Schwimmer JB, Van Natta ML, et al. Effect of vitamin $\mathrm{E}$ or metformin for treatment of nonalcoholic fatty liver disease in children and adolescents: the TONIC randomized controlled trial. JAMA. 2011;305:1659-68.

76. Uygun A, Kadayfci A, Isik AT, et al. Metformin in the treatment of patients with non-alcoholic steatohepatitis. Aliment Pharmacol Ther. 2004;19:537-544.

77. Nar A, Gedik O. The effect of metformin on leptin in obese patients with type 2 diabetes mellitus and nonalcoholic fatty liver disease. Acta Diabetol. 2009;46:113-8.

78. Haukeland JW, Konopski Z, Eggesbo HB, et al. Metformin in patients with non-alcoholic fatty liver disease: a randomized, controlled trial. Scand J Gastroenterol. 2009;44:853-60.

79. Sofer E, Boaz M, Matas Z, et al. Treatment with insulin sensitizer metformin improves arterial properties, metabolic parameters, and liver function in patients with nonalcoholic fatty liver disease: a randomized, placebo-controlled trial. Metabolism. 2011;60:1278-84.

80. Angelico F, Burattin M, Alessandri C, et al. Drugs improving insulin resistance for non-alcoholic fatty liver disease and/or non-alcoholic steatohepatitis. Cochrane Database Syst Rev. 2007;CD005166.

81. Socha P, Horvath A, Vajro P, et al. Pharmacological interventions for nonalcoholic fatty liver disease in adults and in children: a systematic review. J Pediatr Gastroenterol Nutr. 2009;48:587-96.

82. Rakoski MO, Singal AG, Rogers MA, et al. Meta-analysis: insulin sensitizers for the treatment of non-alcoholic steatohepatitis. Aliment Pharmacol Ther. 2010;32:1211-21.

83. Shyangdan D, Clar C, Ghouri N, et al. Insulin sensitisers in the treatment of non-alcoholic fatty liver disease: a systematic review. Health Technol Assess. 2011;15:1-110.

84. Lindor KD, Kowdley KV, Heathcote EJ, et al. Ursodeoxycholic acid for treatment of nonalcoholic steatohepatitis: results of a randomized trial. Hepatology. 2004;39:770-8.

85. Leuschner UF, Lindenthal B, Herrmann G, et al. High-dose ursodeoxycholic acid therapy for nonalcoholic steatohepatitis: a double-blind, randomized, placebo-controlled trial. Hepatology. 2010;52:472-9.

86. Musso G, Gambino R, Cassader M, et al. A meta-analysis of randomized trials for the treatment of nonalcoholic fatty liver disease. Hepatology. 2010;52:79-104.

87. Ratziu V, de Ledinghen V, Oberti $\mathrm{F}$, et al. A randomized controlled trial of high-dose ursodesoxycholic acid for nonalcoholic steatohepatitis. J Hepatol. 2011;54:1011-9.

88. Samy W, Hassanian MA. Paraoxonase-1 activity, malondialdehyde and glutathione peroxidase in non-alcoholic fatty liver disease and the effect of atorvastatin. Arab J Gastroenterol. 2011;12:80-5.

89. Foster T, Budoff MJ, Saab S, et al. Atorvastatin and antioxidants for the treatment of nonalcoholic fatty liver disease: the St Francis Heart Study randomized clinical trial. Am J Gastroenterol. 2011;106:71-7.

90. Athyros VG, Mikhailidis DP, Didangelos TP, et al. Effect of multifactorial treatment on non-alcoholic fatty liver disease in metabolic syndrome: a randomised study. Curr Med Res Opin. 2006;22:873-83.

91. Ekstedt M, Franzen LE, Mathiesen UL, et al. Statins in nonalcoholic fatty liver disease and chronically elevated liver enzymes: a histopathological follow-up study. J Hepatol. 2007;47:135-41.

92. Hyogo H, Tazuma S, Arihiro K, et al. Efficacy of atorvastatin for the treatment of nonalcoholic steatohepatitis with dyslipidemia. Metabolism. 2008;57:1711-8.
93. Park H, Hasegawa G, Shima T, et al. The fatty acid composition of plasma cholesteryl esters and estimated desaturase activities in patients with nonalcoholic fatty liver disease and the effect of long-term ezetimibe therapy on these levels. Clin Chim Acta. 2010;411:1735-40.

94. Yoneda M, Fujita K, Nozaki Y, et al. Efficacy of ezetimibe for the treatment of non-alcoholic steatohepatitis: an open-label, pilot study. Hepatol Res. 2010;40:566-73.

95. Enjoji M, Machida K, Kohjima M, et al. NPC1L1 inhibitor ezetimibe is a reliable therapeutic agent for non-obese patients with nonalcoholic fatty liver disease. Lipids Health Dis. 2010;9:29.

96. Yokohama S, Yoneda M, Haneda M, et al. Therapeutic efficacy of an angiotensin II receptor antagonist in patients with nonalcoholic steatohepatitis. Hepatology. 2004;40:1222-5.

97. Yokohama S, Tokusashi Y, Nakamura K, et al. Inhibitory effect of angiotensin II receptor antagonist on hepatic stellate cell activation in non-alcoholic steatohepatitis. World J Gastroenterol. 2006;12:322-6.

98. Georgescu EF, Ionescu R, Niculescu M, et al. Angiotensin-receptor blockers as therapy for mild-to-moderate hypertensionassociated non-alcoholic steatohepatitis. World J Gastroenterol. 2009;15:942-54.

99. Zein CO, Yerian LM, Gogate P, et al. Pentoxifylline improves nonalcoholic steatohepatitis: a randomized placebo-controlled trial. Hepatology. 2011;54:1610-9.

100. Van Wagner LB, Koppe SW, Brunt EM, et al. Pentoxifylline for the treatment of non-alcoholic steatohepatitis: a randomized controlled trial. Ann Hepatol. 2011;10:277-86.

101. Li W, Zheng L, Sheng C, et al. Systematic review on the treatment of pentoxifylline in patients with non-alcoholic fatty liver disease. Lipids Health Dis. 2011;10:49.

102. Abdelmalek MF, Sanderson SO, Angulo P, et al. Betaine for nonalcoholic fatty liver disease: results of a randomized placebo-controlled trial. Hepatology. 2009;50:1818-26.

103. Charlton MR, Burns JM, Pedersen RA, et al. Frequency and outcomes of liver transplantation for nonalcoholic steatohepatitis in the US. Gastroenterology. 2011;141:1249-53.

104. Yalamanchili K, Saadeh S, Klintmalm GB, et al. Nonalcoholic fatty liver disease after liver transplantation for cryptogenic cirrhosis or nonalcoholic fatty liver disease. Liver Transpl. 2010;16:431-9.

105. Bhagat V, Mindikoglu AL, Nudo CG, et al. Outcomes of liver transplantation in patients with cirrhosis due to nonalcoholic steatohepatitis versus patients with cirrhosis due to alcoholic liver disease. Liver Transpl. 2009;15:1814-20.

106. Dureja P, Mellinger J, Agni R, et al. NAFLD recurrence in liver transplant recipients. Transplantation. 2011;91:684-9.

107. Malik SM, deVera ME, Fontes P, et al. Outcome after liver transplantation for NASH cirrhosis. Am J Transplant. 2009;9:782-93.

108. Feldstein AE, Charatcharoenwitthaya P, Treeprasertsuk S, et al. The natural history of non-alcoholic fatty liver disease in children: a follow-up study for up to 20 years. Gut. 2009;58:1538-44.

109. Fujita N, Miyachi H, Tanaka H, et al. Iron overload is associated with hepatic oxidative damage to DNA in nonalcoholic steatohepatitis. Cancer Epidemiol Biomark Prev. 2009;18:424-32.

110. Facchini FS, Hua NW, Stoohs RA. Effect of iron depletion in carbohydrate-intolerant patients with clinical evidence of nonalcoholic fatty liver disease. Gastroenterology. 2002;122:931-9.

111. Fargion S, Dongiovanni P, Guzzo A, et al. Iron and insulin resistance. Aliment Pharmacol Ther. 2005;22(Suppl 2):61-3.

112. Sumida Y, Kanemasa K, Fukumoto K, et al. Effect of iron reduction by phlebotomy in Japanese patients with nonalcoholic steatohepatitis: a pilot study. Hepatol Res. 2006;36:315-21. 
113. Riquelme A, Soza A, Nazal L, et al. Histological resolution of steatohepatitis after iron depletion. Dig Dis Sci. 2004;49:1012-5.

114. Valenti L, Fracanzani AL, Dongiovanni P, et al. Iron depletion by phlebotomy improves insulin resistance in patients with nonalcoholic fatty liver disease and hyperferritinemia: evidence from a case-control study. Am J Gastroenterol. 2007;102: 1251-8.

115. Adams LA, Lymp JF, St Sauver J, et al. The natural history of nonalcoholic fatty liver disease: a population-based cohort study. Gastroenterology. 2005;129:113-21.

116. Ekstedt M, Franzen LE, Mathiesen UL, et al. Long-term followup of patients with NAFLD and elevated liver enzymes. Hepatology. 2006;44:865-73.

117. Rafiq N, Bai C, Fang Y, et al. Long-term follow-up of patients with nonalcoholic fatty liver. Clin Gastroenterol Hepatol. 2009;7:234-8.

118. Soderberg C, Stal P, Askling J, et al. Decreased survival of subjects with elevated liver function tests during a 28-year follow-up. Hepatology. 2010;51:595-602.

119. Sorensen HT, Mellemkjaer L, Jepsen P, et al. Risk of cancer in patients hospitalized with fatty liver: a Danish cohort study. J Clin Gastroenterol. 2003;36:356-9.

120. Teli MR, James OF, Burt AD, et al. The natural history of nonalcoholic fatty liver: a follow-up study. Hepatology. 1995;22:1714-9.

121. Dam-Larsen S, Becker U, Franzmann MB, et al. Final results of a long-term, clinical follow-up in fatty liver patients. Scand $\mathbf{J}$ Gastroenterol. 2009;44:1236-43.

122. Evans CD, Oien KA, MacSween RN, et al. Non-alcoholic steatohepatitis: a common cause of progressive chronic liver injury? J Clin Pathol. 2002;55:689-92.

123. Targher G, Bertolini L, Poli F, et al. Nonalcoholic fatty liver disease and risk of future cardiovascular events among type 2 diabetic patients. Diabetes. 2005;54:3541-6.

124. Hamaguchi M, Kojima T, Takeda N, et al. Nonalcoholic fatty liver disease is a novel predictor of cardiovascular disease. World J Gastroenterol. 2007;13:1579-84.
125. Musso G, Gambino R, Cassader M, et al. Meta-analysis: natural history of non-alcoholic fatty liver disease (NAFLD) and diagnostic accuracy of non-invasive tests for liver disease severity. Ann Med. 2011;43:617-49.

126. Bugianesi E, Leone N, Vanni E, et al. Expanding the natural history of nonalcoholic steatohepatitis: from cryptogenic cirrhosis to hepatocellular carcinoma. Gastroenterology. 2002;123:134-40.

127. Hashizume H, Sato K, Takagi H, et al. Primary liver cancers with nonalcoholic steatohepatitis. Eur J Gastroenterol Hepatol. 2007;19:827-34.

128. El-Serag HB, Tran T, Everhart JE. Diabetes increases the risk of chronic liver disease and hepatocellular carcinoma. Gastroenterology. 2004;126:460-8.

129. Tokushige K, Hashimoto E, Yatsuji S, et al. Prospective study of hepatocellular carcinoma in nonalcoholic steatohepatitis in comparison with hepatocellular carcinoma caused by chronic hepatitis C. J Gastroenterol. 2010;45:960-7.

130. Kodama K, Tojjar D, Yamada S, et al. Ethnic differences in the relationship between insulin sensitivity and insulin response: a systematic review and meta-analysis. Diabetes Care. 2013;36:1789-96.

131. Bhatt SP, Misra A, Sharma M, et al. Ala/Ala genotype of Pro12Ala polymorphism in the peroxisome proliferator-activated receptor-gamma2 gene is associated with obesity and insulin resistance in Asian Indians. Diabetes Technol Ther. 2012;14:828-34.

132. Thameem F, Puppala S, Schneider J, et al. The Gly(972)Arg variant of human IRS1 gene is associated with variation in glomerular filtration rate likely through impaired insulin receptor signaling. Diabetes. 2012;61:2385-93.

133. Mostafa SA, Davies MJ, Morris DH, et al. The association of the triglyceride-to-HDL cholesterol ratio with insulin resistance in White European and South Asian men and women. PLoS One. 2012;7:e50931. 